\title{
Women's Cardiac Health in 2020: A Systematic Review
}

\author{
Fabiola B. Sozzi ${ }^{1}$ Marta Belmonte ${ }^{1}$ Marco Schiavone ${ }^{1} \quad$ Ciro Canetta $^{2} \quad$ Rakesh Gupta $^{3} \quad$ Francesco Blasi $^{1}$
}

${ }^{1}$ Fondazione IRCCS Ca' Granda Ospedale Maggiore Policlinico, Internal Medicine Department, Respiratory Unit, Cystic Fibrosis Adult Center, and Cardiovascular Unit, Milan, Italy

2 Internal Medicine Unit, Crema Hospital, Lombardy, Italy

${ }^{3}$ JROP Institute of Echocardiography, Delhi, India

J Card Crit Care:2020;4:104-110
Address for correspondence Fabiola B. Sozzi, MD, PhD, Ospedale Maggiore Policlinico, IRCCS, Cà Granda, via F. Sforza 35, 20122, Milan, Italy (e-mail: fabiola_sozzi@yahoo.it).

\begin{abstract}
Although substantial progress has been made toward improving gender- and sex-specific cardiovascular disease (CVD) management and outcomes, contemporary reports indicate a persistent knowledge gap with regard to optimal risk-stratification and management in female cardiac heart disease (CHD) patients. Prominent patient and system delays in diagnosing CHD are, in part, due to the limited awareness for the latent CVD risk in women, a lack of sex-specific thresholds within clinical guidelines, and subsequent limited performance of contemporary diagnostic approaches in women. Several traditional risk factors for CHD affect both women and men. But other factors can play a bigger role in the development of heart disease in women. In addition, little is known about the influence of socioenvironmental and contextual factors on gender-specific disease manifestation and outcomes. It is imperative that we understand the mechanisms that contribute to worsening risk factors profiles in young women to reduce future atherosclerotic CVD morbidity and mortality. This comprehensive review focuses on the novel aspects of car-

\section{Keywords}

- cardiovascular disease

- COVID-19

- women's cardiac health diovascular health in women and sex differences as they relate to clinical practice and prevention, diagnosis, and treatment of CVD. Increased recognition of the prevalence of traditional cardiovascular risk factors and their differential impact in women, as well as emerging nontraditional risk factors unique to or more common in women, contribute to new understanding mechanisms, leading to worsening outcome for women.
\end{abstract}

\section{Cardiovascular Health}

Ideal cardiovascular health, which is one of the "Strategic Impact Goals" for 2020 introduced by the American Heart Association (AHA) in 2011, is defined by both the absence of clinically manifest cardiovascular diseases (CVDs) and the presence of optimal levels of 7 metrics, the so-called Life's simple 7. ${ }^{1,2}$ The latter includes health behavior (not smoking, having a healthy diet pattern, sufficient physical activity) and health factors (normal body mass index [BMI], and normal levels of total cholesterol, blood pressure, and fasting blood glucose) in the absence of drug treatment. The organizational

published online October 27, 2020
DOI https://doi.org/ 10.1055/s-0040-1718503 ISSN 2457-0206. priority to improve by 2020 cardiovascular health by $20 \%$ is supported by data showing worldwide a strong inverse, stepwise association of ideal metrics with all-cause mortality, cardiovascular (CVD) risk, and mortality. In particular, it has been shown that globally cardiovascular health is associated with a decrease in the incidence of heart failure $(\mathrm{HF})^{3}$, better prognosis after myocardial infarction (MI), ${ }_{4}^{4}$ lower prevalence of aortic sclerosis and stenosis, ${ }^{5}$ and lower risk of atrial fibrillation (AF). ${ }^{6}$ Moreover, lower rates of physical functional impairment, frailty, cognitive decline, and depression have been reported. ${ }^{7}$ These associations lead to a relevant decrease in the health care cost. ${ }^{8}$ 
The achievement of ideal cardiovascular health implies a deep knowledge of gender-related differences in incidence, pathophysiology, diagnosis, and treatment for both primary and secondary prevention of CVD, which are relevant issues for women.

CVD is the leading cause of death worldwide, accounting for $47 \%$ of deaths in females and 39\% in males in European Society of Cardiology (ESC) member countries ( 2.2 million deaths in females and 1.9 million deaths in males in the most recent year of available data in Europe). ${ }^{9}$ Similar rates of mortality have been shown also in the U.S. population (219.4 per 100,000 in 2016). ${ }^{1}$ Cardiac heart disease (CHD) is the leading cause of mortality in both Europe (38\% of cardiovascular deaths in females and $44 \%$ in males) and the United States (43.2\%). ${ }^{1,9}$

The longer lifespan of women versus men will result in a prevalently-female elderly population being seen for CVD, mainly CHD and HF, by health care providers, who at the moment are not trained enough to recognize and treat special aspects of CHD in women. There are, indeed, both sex (biological) and gender (sociocultural) differences in CVD and its outcomes, but robust evidence on this topic is lacking. In fact, women have been largely underrepresented in clinical trials.

It has been estimated that in cardiovascular clinical trials published from 1997 to 2009, women accounted only for onethird of the total population enrolled, with even lower representation when considering specific CVD (e.g., chronic and acute coronary syndromes, HF). ${ }^{10}$ In the last decade, a trend toward a greater enrollment of women in clinical trials has been shown, with an average percentage of women enrolled of $38.2 \%$, with the lowest prevalence in interventional trials. ${ }^{11}$

Fortunately, in 2016, the National Institutes of Health made the inclusion of both sexes in cell and animal studies mandatory. ${ }^{12}$ Large-scale studies investigating differences in the diagnosis and treatment of CVD in women are ongoing and will hopefully lead to the definition of disease patterns and efficacious therapies unique to women.

Women have significantly smaller epicardial coronary arteries than men, even after adjustment for age, body habitus, and left ventricular mass. Although the exact mechanisms are lacking, the smaller diameter of female epicardial coronary arteries together with their higher baseline myocardial blood flow have been suggested to result in a significant increase in endothelial shear stress conditions in women. Given that low endothelial shear stress has been associated with focal lipid accumulation, pathologic remodeling, and plaque instability, it has been hypothesized that higher shear stress conditions in female coronary arteries may contribute to sex differences in susceptibility to CHD. ${ }^{13}$ These sex differences might be particularly relevant during premenopausal ages owing to estrogen-dependent effects on endothelial mediators such as nitric oxide, prostaglandins, and endothelium-derived hyperpolarizing factor.

Ischemic heart disease includes disease originating in the coronary arteries, in the microcirculation, and from an imbalance in myocardial oxygen supply and demand. In women, there is a lower prevalence of anatomically obstructive coronary artery disease yet greater rates of myocardial ischemia and associated mortality compared with similarly aged males. The Women's Ischemia Syndrome Evaluation (WISE) study and other related studies have implicated abnormal coronary reactivity, microvascular dysfunction, and plaque erosion/distal microembolization as causative of female-specific ischemic disease pathophysiology. Women with ischemic heart disease have a persistent suboptimal treatment pattern, higher mortality, and poorer CVD outcome compared with men. ${ }^{14}$

\section{Health Behavior}

\section{Smoking}

The mean prevalence of tobacco uses in 2014 was approximately $21 \%$ in males and females aged $\geq 15$ years in Europe and in the United States, with slight regional and age group differences. ${ }^{1,9}$ Although regular daily smoking is more common in men than in women (17.5\% of males and $13.5 \%$ of females are current smokers), female smokers have a $25 \%$ greater risk of CHD than male ones. ${ }^{15}$

The contribution of smoking to overall risk of atherosclerotic disease in women is influenced by age, smoking habits and cessation, and oral contraceptive use. The relative increase in risk due to smoking is generally greater in the subset of women with the lower prevalence of other traditional risk factors for MI. In particular, the increase in relative risk (RR) among female smokers versus nonsmokers is 13-fold among those aged 25 to 29 years and 5-fold among those aged 45 to 49 years. However, the absolute increase in risk due to smoking is usually greater among those whose baseline risk is higher, that is, among older women. ${ }^{16}$ The use of oral contraceptives has a synergistic effect on CVD risk, likely due to prothrombotic effects, with third-generation hormonal contraceptives posing less risk than prior and fourth-generation formulation. ${ }^{17}$

Overall, the increasing awareness of the detrimental effects of smoking, the approval of restrictive laws, and the availability of a wide variety of methods to quit smoking have led to a decline in smoking worldwide, with a stronger decline in males. The lower cessation rates of smoking in women may account for the less favorable trend in smoking prevalence in women with respect to men. When approaching to quitting smoking, women report specific barriers, among which the most common are fear of weight gain, insufficient motivation and confidence in their ability to succeed, and distress during the cessation period. ${ }^{18}$ Moreover, nicotine replacement therapy is not as successful in women as in men, probably because women seem to have higher "behavioral" dependence on smoking, in opposite to the greater "pharmacological" dependence of men. Uniquely to women, the menstrual cycle has been shown to affect the success rate of smoking cessation therapy. Higher relapse rates were indeed observed in women during the follicular phase of cycle or in women with premenstrual symptoms, in particular with craving and anger.

Cessation of smoking decreases the risk for CVD to that of nonsmokers after approximately 10 years. ${ }^{19}$ However, data regarding the long-term effects of other increasingly common forms of tobacco use (e.g., electronic cigarettes) are lacking. 


\section{Diet}

Given the limitations in evaluating the impact of solely nutrition on CVD risk due to the influence of healthy lifestyle and imprecise measurement of dietary intake, it has been shown that healthy diet (e.g., DASH [Dietary Approaches to Stop Hypertension] type or Mediterranean type) reduces population-adjusted cardiometabolic deaths. ${ }^{20}$ Overall, the prevalence of a poor diet has decreased (from 50.3\% to $41.0 \%$ in the United States), ${ }^{21}$ especially among women, who on average consume more fruit and vegetables than men. However, economic and sociocultural factors play a crucial role in gender-related and regional differences in nutrition.

In developed countries, women tend to consume foods with added sugars, sweets, and bakery desserts; whereas men consume greater amount of meat and alcohol. ${ }^{22}$

Local sociocultural beliefs influence the female body image, with overweight being disregarded in western countries and considered to be associated with high social status in Middle Eastern countries. Therefore, western countries women spending more time, effort, and money to obtain the ideal thinner shape.

Conversely, in developing countries, undernutrition affects cardiovascular health.

Undernutrition during pregnancy has been associated with increased risk of cardiovascular and metabolic disease in later life, presumably through epigenetic modifications. ${ }^{23}$ When undernutrition in early life is followed by overnutrition in adult life, the risk of adult chronic disease is increased further, as postulated for the first time in the Barker Hypothesis in the early 1980s. ${ }^{24}$ The period of life that is most sensible to the dietary mismatch is adolescence. Therefore, promoting healthy lifestyles and avoiding dietary excesses is crucial to preserve cardiovascular health.

\section{Physical Activity}

The prevalence of physical inactivity is 31\% among adults aged $\geq 18$ years in Europe and $26.9 \%$ in the United States, with higher rates in women, in high-income countries, and in the elderly. ${ }^{1,9}$

Physical inactivity is included in the criteria to define a woman "at risk" for CVD, with prolonged sitting time being independently associated with high CVD and all-cause mortality risk. ${ }^{19,20}$ To quantify exercise capacity, also known as physical fitness, a nomogram for asymptomatic women is available. ${ }^{25}$ However, occupational, transportation, and domestic domains of physical activity are overlooked since they are not included in traditional physical activity surveys, which account only for leisure-time activities. Transportation activity and high domestic work showed a protective effect on CVD risk. ${ }^{26,27}$ The benefit of exercise is evident also in pregnant women, with aerobic exercise during pregnancy being associated with a significantly lower risk of gestational hypertensive disorders (RR: $0.70 ; 95 \%$ confidence interval: $0.53-0.83$ ). ${ }^{28}$

\section{Body Mass Index}

Worldwide, the average BMI for men and women has steadily increased over the last decades. The mean age-adjusted prevalence of obesity, defined as a BMI $>30 \mathrm{~kg} / \mathrm{m}^{2}$, is higher in females than in men (in the United States $41.1 \%$ of females and $37.9 \%$ of males; in Europe $22.8 \%$ in females and $22.3 \%$ in males), ${ }^{1,9}$ and $7.7 \%$ had class III obesity (5.6\% of males and $9.7 \%$ of females $).{ }^{29}$ This is particularly relevant in developing countries, with higher obesity rates occurring earlier (at the age of $\geq 25$ years), whereas in middle-high income countries, obesity tends to be more common in males.

Obesity is associated with an increased lifetime risk of CVD and increased prevalence of type 2 diabetes mellitus (DM), hypertension, dyslipidemia, sleep-disordered breathing, venous thromboembolism, AF, and dementia. ${ }^{30,31}$ CVD appears to be related to the pattern of obesity, that is, visceral obesity, which forms the typical "android phenotype." Peripheral adiposity, with subcutaneous adipose tissue accumulation in both abdominal (waist) and gluteofemoral area, typical of premenopause women, seems to be protective. The shift in the body fat distribution toward a more central/android pattern of postmenopause women could explain the steep increase in CVD risk in the postmenopause period, which equals the one of men of the same age. However, recent data suggest that obesity could be not an independent risk factor for CVD, but it exerts a negative impact because of its association with major traditional risk factors, especially with DM, physical inactivity, and hypertension. ${ }^{32,33}$ Bariatric surgery, which is becoming increasingly popular due to greater weight loss and longer maintenance of lost weight than lifestyle intervention, leads to fewer CVD events probably not only for substantial weight loss, but also for remission of DM, hypertension, and dyslipidemia. ${ }^{34}$

\section{Health Factors}

\section{Blood Cholesterol}

Cholesterol is a major primary causal risk factor for the development of CHD. It is thus a major target of risk reduction programs, in which statin therapy in primary prevention can produce a $15 \%$ reduction in risk of vascular death for each $1-\mathrm{mmol} / \mathrm{L}$ reduction of low-density lipoprotein (LDL) cholesterol. ${ }^{35-37}$ The estimated prevalence of elevated serum total cholesterol has decreased to an average of $11.7 \%$ in the United States, with higher rates in females (12.4\%) than males (10.7\%) and lower rates in black males than other racial groups. This is mostly due to greater uptake of cholesterol-lowering medications rather than adoption of healthy lifestyle. ${ }^{38}$ Notably, lipid profiles are different between premenopausal women and men. The former have higher levels and larger high-density lipoprotein (HDL) particles, ${ }^{39}$ with greater efflux capacity, ${ }^{40}$ lower LDL cholesterol, total plasma triglyceride, and triglyceride concentrations compared with age-matched men. This favorable lipid profile, mainly due to sex-related difference in fat distribution and hormonal influence on lipid metabolism, changes after menopause, with a decrease in HDL and an increase in LDL; whether this change is due to menopause-related hormonal changes or aging is debated. ${ }^{41}$ 


\section{Blood Glucose}

Untreated fasting glucose level $<100 \mathrm{mg} / \mathrm{dL}$ for children and adults is one of seven components of ideal cardiovascular health since the increase of glucose level above the mentioned cutoff (both pre-DM and DM) is associated with an increase of CVD, particularly CHD, and all-mortality risk. Although DM is more common in men rather than in women (in Europe averaged prevalence of 6.8\%; in the United States $9.4 \%$ in white males and $7.3 \%$ in white females, with peaks of $14.7 \%$ in black males and $13.4 \%$ in black females), it is more detrimental for female health. Women with type 2 DM have a twofold excess CHD risk compared with men and a threefold excess fatal CHD risk compared with nondiabetic women, with a higher adjusted hazard ratio of fatal CHD compared with men with DM. A possible explanation could be that DM in women is associated with greater impairment of endothelium-dependent vasodilatation, worse atherogenic dyslipidemia, prothrombotic coagulation profile, higher metabolic syndrome prevalence, and other CHD risk factors that have a synergic interaction. ${ }^{42}$ In women only, abdominal adiposity, which is closely related to insulin resistance, has appeared to be strongly associated with cardiovascular mortality.

Other postulated contributors to the increased CHD mortality risk in women with DM are sex-related disparities in application of the guideline-recommended risk factor management strategies and in efficacy of treatment.

Women with DM are less likely to receive appropriate care both in outpatient clinic settings ${ }^{43}$ and after acute coronary syndromes. ${ }^{44}$ Revascularization rates of CHD with angioplasty and coronary artery bypass surgery are lower in women than men with DM, all contributing to more CHD-related complications in diabetic women. ${ }^{45}$

Unique to women of reproductive age, polycystic ovary syndrome and gestational DM pose them at increased risk of developing DM, and should be considered when collecting the medical history of female patients. These diseases give the opportunity to analyze the interaction of sex steroid hormones and insulin resistance before DM becoming clinically evident. Insulin resistance is a key feature of polycystic ovary syndrome and treatments improving insulin sensitivity can lower androgens and restore ovulatory cycles, suggesting that high bioavailable or unbound testosterone may be harmful for women and associated with increased CVD risk factors. ${ }^{46}$

A history of gestational DM doubles the risk of DM in the 4-months postpartum and remains a life-long risk factor for DM and CVD. ${ }^{47}$ Moreover, it has been shown that gestational DM could be a marker for early atherosclerosis independent of prepregnancy obesity among women who have not developed type $2 \mathrm{DM}$ or the metabolic syndrome. ${ }^{48}$ It is considered as an "at-risk" criterion for women according to guidelines and implies, thus, the application of specific screening programs and therapeutic lifestyle changes.

\section{Blood Pressure}

Hypertension is the most common modifiable risk factor for CVD in both sexes, affecting around one-third of population globally. Women have a higher prevalence of hypertension than men after the age of 60,49 although younger women taking oral contraceptives have blood pressure values 7 to $8 \mathrm{~mm} \mathrm{Hg}$ higher than age-matched women on average. The highest prevalence of hypertension in the world is in blacks in the United States (57.6\% among males and 53.2\% among females), who are more likely to have resistant hypertension (19\%) than whites $(13.5 \%)$ or Hispanics (11.2\%). ${ }^{50}$

Worldwide, women are more aware of their diagnosis of hypertension compared with men (72\% women vs. $62 \%$ men in high-income countries, $36 \%$ women vs. $22 \%$ men in low-income countries). ${ }^{51}$ Hypertensive women more likely receive antihypertensive medications (52\% women vs. $49 \%$ men in high-income countries, $28 \%$ women vs. $23 \%$ men in low-income countries). ${ }^{52}$ Control (defined as systolic blood pressure $<140 \mathrm{~mm} \mathrm{Hg}$ and diastolic blood pressure < 90 $\mathrm{mm} \mathrm{Hg}$ ) rates are higher in women than men under the age of 60 , whereas the proportion reverses after the age of 60 . Overall, middle-/low-income countries have lower rates of awareness and treatment mainly due to limited access to health care and medications..$^{53}$

Moreover, women are more likely to experience adverse effects associated with antihypertensives than men. ${ }^{49}$ The risk of angiotensin-converting enzyme inhibitor induced cough among females is threefold greater, as well as peripheral edema with calcium channel blockers and low serum levels of sodium and potassium with thiazide diuretic therapy. Interestingly, a higher rate of hypotension has been seen in women taking antihypertensive drugs compared with men, ${ }^{54}$ probably due to antihypertensive medication titration based on in-office blood pressure readings, which tend to be higher in women compared with men. In fact, white coat hypertension is more common among women compared with men, with a prevalence of $43 \%$ versus $34 \%$, respectively, in the United States and similar trends worldwide. However, hypertension is the risk factor that has the largest independent impact on CVD mortality among women. Optimal blood pressure control leads to a reduction in CVD mortality by $38.0 \%$ in females compared with $30.4 \%$ in males. ${ }^{55}$

Therefore, guidelines strongly encourage a comprehensive assessment and treatment of hypertension in women, considering also sex-specific forms of hypertension such as pregnancy-related hypertensive disorders.

Hypertension during pregnancy, which ranges from gestational hypertension to eclampsia, has been associated with CVD (mainly hypertension, metabolic syndrome, and $\mathrm{AF})^{56}$ and increased mortality later in life. ${ }^{57}$ Treatment of hypertension during pregnancy is complicated by the limited range of antihypertensive drugs that do not cross the placenta and by uncertainty about treatment thresholds and goals. Since the prevalence of hypertension during pregnancy is increasing in both developed and developing countries, it is expected that more and more women will require medical attention for cardiovascular implications during follow-up. 


\section{Mental Stress and Depression in Women: Understanding the Gender Gap}

Research suggests that depression increases the likelihood of developing CHD and stroke. The issue is important to consider for women in particular because they are nearly twice as likely as men to be diagnosed with depression. Depression can occur at any age. Some mood changes and depressed feelings occur with normal hormonal changes. Indeed, it is common during times of hormonal fluctuations. Several factors may increase a woman's risk of depression. The addition of other biological factors, inherited traits, and personal life circumstances and experiences are associated with a higher risk to develop it. Women who feel the strain of working in high-pressure jobs have, overall, a 40\% higher risk of CHD. Research has found a stronger link between depression and anxiety disorders, and CHD in women than in men. ${ }^{58,59}$ Women are more likely than men to report physical and emotional symptoms of stress. Many women play significant roles both in the home and in the workforce, making them more susceptible to stress than men. How much we earn and how well we live also are closely linked to overall health. In many cases, women have lower socioeconomic status than men, and those with lower socioeconomic backgrounds and lower levels of education also have higher rates of CHD than educated women in the higher income bracket. Females have statistically significantly higher rates of work-related stress depression and anxiety compared with the average for all persons. This is evident in the age range of 25 to 54 years. Males did not have statistically significant rates compared with all persons. The reasons cited as causes of work-related stress are also consistent over time with workload, lack of managerial support, and organizational change as the primary causative factors. A study on the work of an international collaboration of physicians from the United States and Europe ${ }^{60}$ evaluated 1,750 patients with Takotsubo cardiomyopathy. Interestingly, $90 \%$ of these cases occurred in women, and the women in this study were an average of 67 years old. The most common triggers of stress cardiomyopathy were physical (such as lung problems or infections), and the next most common cause was an emotional "shock." But in a substantial proportion of patients, there was no trigger that could be identified. Compared with people who had experienced a "typical" heart attack, patients with Takotsubo cardiomyopathy were almost twice as likely to have a neurological or psychiatric disorder. And in contrast to the commonly-held belief among doctors that Takotsubo cardiomyopathy is less serious than other forms of heart attack, the rates of death in the hospital between Takotsubo cardiomyopathy and more "traditional" heart attacks were similar.

\section{Prevention of Cardiovascular Disease in Women}

Despite substantial progress in the awareness of CVD among women and an increasing amount of literature in this field, disparity, defined as a difference in treatment provided to members of ethnic or racial groups that is not justified by health condition differences or treatment preferences, still exists (-Fig. 1).

Not only guidelines keep on recommending risk scores (e.g., Framingham risk score) that are often inadequate to appropriately estimate CVD risk in women, but they are not always applied in the management of female patients, and, when implemented in clinical practice, but they also do not always lead to effective treatment. The algorithm for risk classification in women, proposed in 2011, could help to overcome discrepancies, moving from "evidence-based" to "effectiveness-based" guidelines ${ }^{61}$ It consists in a risk stratification into three categories, "at high risk," "at risk," and "at optimal risk," including consideration of factors neglected from previous risk scores (e.g., medical and lifestyle history, family history of CVD, markers of preclinical disease), to make decisions about the intensity of preventive therapy. Besides the traditional CVD risk factors, other nontraditional risks that are unique to women should be thoroughly assessed. Pregnancy has gained greater attention since it can be considered as a unique "cardiovascular and metabolic stress," which can unmask preclinical or preexisting CVD. Therefore, detailed obstetric and gynecologic histories should be obtained at least at the first visit. Moreover, novel CVD risk biomarkers (e.g., hsCRP [high-sensitivity C-reactive protein] or advanced lipid testing) and imaging technologies (e.g., coronary calcium scoring assessment) have been proposed in risk assessment and in guidance of appropriate preventive interventions, but their role is still debated. Physicians should carefully assess barriers common among women that hinder adherence to CVD prevention recommendations, for example, family and caretaking responsibilities, stress, sleep deprivation, fatigue, and lack of personal time. To achieve the targets

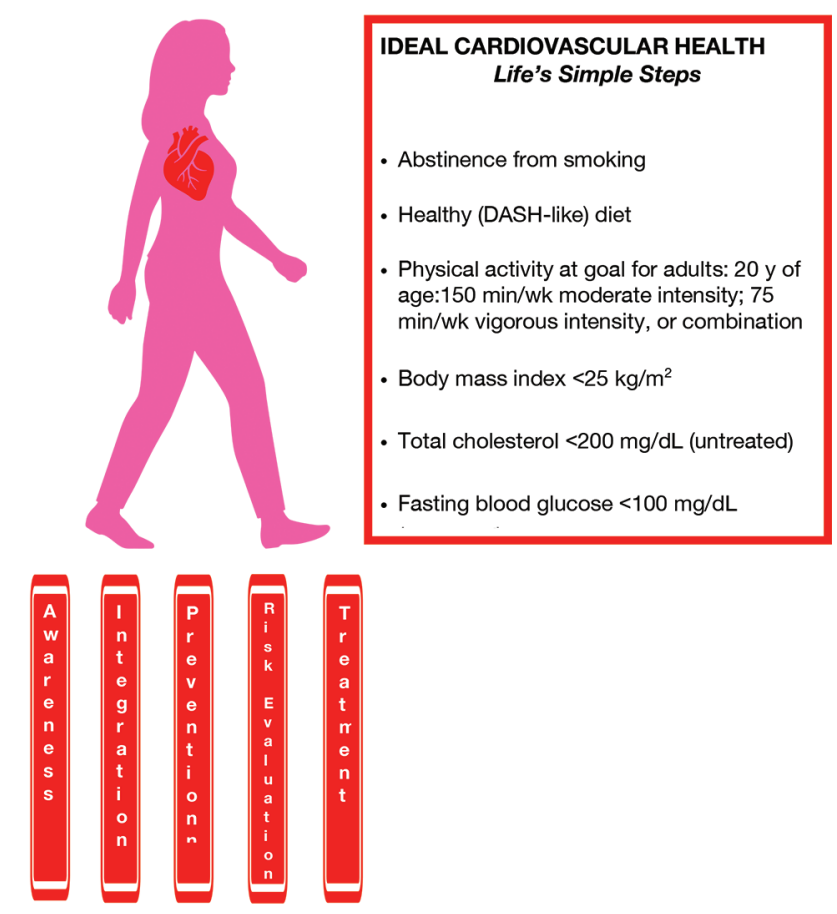

Fig. 1 Ideal cardiovascular health parameters in women. 
of intervention, multifaceted, interactive, and incorporate decision systems and feedback have been shown to be successful in increasing awareness and improving health and lifestyles for women and their family members.

\section{Conclusions}

Cardiovascular health in women, which is effective in the prevention of CVD, should be promoted worldwide, with great efforts in overcoming the still existing disparity. The goal set by the AHA for 2020 could be achieved by greater educational information about the importance of CVD in women by the definition of diagnostic and treatment algorithms peculiar to women, greater socioeconomic integration, and improved interdisciplinary and cooperative care.

\section{Conflict of Interest}

None declared.

\section{References}

1 Virani SS, Alonso A, Benjamin EJ, et al; American Heart Association Council on Epidemiology and Prevention Statistics Committee and Stroke Statistics Subcommittee. Heart Disease and Stroke Statistics-2020 Update: a report from the American Heart Association. Circulation 2020;141(9):e139-e596

2 Lloyd-Jones DM, Hong Y, Labarthe D, et al; American Heart Association Strategic Planning Task Force and Statistics Committee. Defining and setting national goals for cardiovascular health promotion and disease reduction: the American Heart Association's strategic Impact Goal through 2020 and beyond. Circulation 2010;121(4):586-613

3 Folsom AR, Shah AM, Lutsey PL, et al. American Heart Association's Life's Simple 7: avoiding heart failure and preserving cardiac structure and function. Am J Med 2015;128(9): 970-6.e2

4 Mok Y, Sang Y, Ballew SH, et al. American Heart Association's Life's Simple 7 at middle age and prognosis after myocardial infarction in later life. J Am Heart Assoc 2018;7(4):e007658

5 Sengeløv M, Cheng S, Biering-Sørensen T, et al. Ideal cardiovascular health and the prevalence and severity of aortic stenosis in elderly patients. J Am Heart Assoc 2018;7(3):e007234

6 Garg PK, O'Neal WT, Chen LY, et al. American Heart Association's Life Simple 7 and risk of atrial fibrillation in a population without known cardiovascular disease: the ARIC (Atherosclerosis Risk in Communities) Study. J Am Heart Assoc 2018; 7(8):e008424

7 Shay CM, Gooding HS, Murillo R, Foraker R. Understanding and improving cardiovascular health: an update on the American Heart Association's concept of cardiovascular health. Prog Cardiovasc Dis 2015;58(1):41-49

8 Osondu CU, Aneni EC, Valero-Elizondo J, et al. Favorable cardiovascular health is associated with lower health care expenditures and resource utilization in a large US employee population: the Baptist Health South Florida Employee Study. Mayo Clin Proc 2017 (e-pub ahead of print). doi:10.1016/j.mayocp.2016.12.026

9 Timmis A, Townsend N, Gale CP, et al; European Society of Cardiology. European Society of Cardiology: Cardiovascular Disease Statistics 2019. Eur Heart J 2020;41(1):12-85

10 Tsang W, Alter DA, Wijeysundera HC, Zhang T, Ko DT. The impact of cardiovascular disease prevalence on women's enrollment in landmark randomized cardiovascular trials: a systematic review. J Gen Intern Med 2012;27(1):93-98
11 Jin X, Chandramouli C, Allocco B, Gong E, Lam CSP, Yan LL. Women's participation in cardiovascular clinical trials from 2010 to 2017. Circulation 2020;141(7):540-548

12 Clayton JA, Collins FS. Policy: NIH to balance sex in cell and animal studies. Nature 2014;509(7500):282-283

13 Patel MB, Bui LP, Kirkeeide RL, Gould KL. Imaging microvascular mysfunction and mechanisms for female-male differences in CAD. JACC Cardiovasc Imaging 2016;9(4):465-482

14 Nicholls SJ, Tuzcu EM, Wolski K, et al. Extent of coronary atherosclerosis and arterial remodelling in women: the NHLBI-sponsored Women's Ischemia Syndrome Evaluation. Cardiovasc Diagn Ther 2018;8(4):405-413

15 Huxley RR, Woodward M. Cigarette smoking as a risk factor for coronary heart disease in women compared with men: a systematic review and meta-analysis of prospective cohort studies. Lancet 2011;378(9799):1297-1305

16 Rosenberg L, Kaufman DW, Helmrich SP, Miller DR, Stolley PD, Shapiro S. Myocardial infarction and cigarette smoking in women younger than 50 years of age. JAMA 1985;253(20):2965-2969

17 Shufelt CL, Bairey Merz CN. Contraceptive hormone use and cardiovascular disease. J Am Coll Cardiol 2009;53(3):221-231

18 Rahmanian SD, Diaz PT, Wewers ME. Tobacco use and cessation among women: research and treatment-related issues. J Womens Health (Larchmt) 2011;20(3):349-357

19 Pirie K, Peto R, Reeves GK, Green J, Beral V; Million Women Study Collaborators. The 21st century hazards of smoking and benefits of stopping: a prospective study of one million women in the UK. Lancet 2013;381(9861):133-141

20 Sanches Machado d'Almeida K, Ronchi Spillere S, Zuchinali P, Corrêa Souza G. Mediterranean diet and other dietary patterns in primary prevention of heart failure and changes in cardiac function markers: a systematic review. Nutrients 2018;10(1):E58

21 Rehm CD, Peñalvo JL, Afshin A, Mozaffarian D. Dietary intake among US adults, 1999-2012. JAMA 2016;315(23):2542-2553

22 Kanter R, Caballero B. Global gender disparities in obesity: a review. Adv Nutr 2012;3(4):491-498

23 Nicoletto SF, Rinaldi A. In the womb's shadow. The theory of prenatal programming as the fetal origin of various adult diseases is increasingly supported by a wealth of evidence. EMBO Rep 2011;12(1):30-34

24 Barker DJ, Mothers, Babies, and Health in Later Life. 2nd ed. Edinburgh: Churchill Livingstone; 1998

25 Chomistek AK, Manson JE, Stefanick ML, et al. Relationship of sedentary behavior and physical activity to incident cardiovascular disease: results from the Women's Health Initiative. J Am Coll Cardiol 2013;61(23):2346-2354

26 Ekelund U, Steene-Johannessen J, Brown WJ, et al; Lancet Physical Activity Series 2 Executive CommitteLancet Sedentary Behaviour Working Group. Does physical activity attenuate, or even eliminate, the detrimental association of sitting time with mortality? A harmonised meta-analysis of data from more than 1 million men and women. Lancet 2016;388(10051):1302-1310

27 Gulati M, Black HR, Shaw LJ, et al. The prognostic value of a nomogram for exercise capacity in women. $\mathrm{N}$ Engl J Med 2005;353(5):468-475

28 Hamer M, Chida Y. Active commuting and cardiovascular risk: a meta-analytic review. Prev Med 2008;46(1):9-13

29 Dhana K, Koolhaas CM, Berghout MA, et al. Physical activity types and life expectancy with and without cardiovascular disease: the Rotterdam Study. J Public Health (Oxf) 2017;39(4):e209-e218

30 Magro-Malosso ER, Saccone G, Di Tommaso M, Roman A, Berghella V. Exercise during pregnancy and risk of gestational 
hypertensive disorders: a systematic review and meta-analysis. Acta Obstet Gynecol Scand 2017;96(8):921-931

31 Hales CM, Fryar CD, Carroll MD, Freedman DS, Ogden CL. Trends in obesity and severe obesity prevalence in US youth and adults by sex and age, 2007-2008 to 2015-2016. JAMA 2018;319(16):1723-1725

32 Eckel N, Meidtner K, Kalle-Uhlmann T, Stefan N, Schulze MB. Metabolically healthy obesity and cardiovascular events: a systematic review and meta-analysis. Eur J Prev Cardiol 2016;23(9):956-966

33 Khan SS, Ning H, Wilkins JT, et al. Association of body mass index with lifetime risk of cardiovascular disease and compression of morbidity. JAMA Cardiol 2018;3(4):280-287

34 Flegal KM, Kit BK, Orpana H, Graubard BI. Association of all-cause mortality with overweight and obesity using standard body mass index categories: a systematic review and meta-analysis. JAMA 2013;309(1):71-82

35 Mihaylova B, Emberson J, Blackwell L, et al; Cholesterol Treatment Trialists' (CTT) Collaborators. The effects of lowering LDL cholesterol with statin therapy in people at low risk of vascular disease: meta-analysis of individual data from 27 randomised trials. Lancet 2012;380(9841):581-590

36 Fulcher J, O'Connell R, Voysey M, et al; Cholesterol Treatment Trialists' (CTT) Collaboration. Efficacy and safety of LDL-lowering therapy among men and women: meta-analysis of individual data from 174,000 participants in 27 randomised trials. Lancet 2015;385(9976) :1397-1405

37 Silverman MG, Ference BA, Im K, et al. Association between lowering LDL-C and cardiovascular risk reduction among different therapeutic interventions: a systematic review and meta-analysis. JAMA 2016;316(12):1289-1297

38 Ford ES, Capewell S. Trends in total and low-density lipoprotein cholesterol among U.S. adults: contributions of changes in dietary fat intake and use of cholesterol-lowering medications. PLoS One 2013;8(5):e65228

39 Wang X, Magkos F, Mittendorfer B. Sex differences in lipid and lipoprotein metabolism: it's not just about sex hormones. J Clin Endocrinol Metab 2011;96(4):885-893

40 Saleheen D, Scott R, Javad S, et al. Association of HDL cholesterol efflux capacity with incident coronary heart disease events: a prospective case-control study. Lancet Diabetes Endocrinol 2015;3(7):507-513

41 Polotsky HN, Polotsky AJ. Metabolic implications of menopause. Semin Reprod Med 2010;28(5):426-434

42 Regensteiner JG, Golden S, Huebschmann AG, et al; American Heart Association Diabetes Committee of the Council on Lifestyle and Cardiometabolic Health, Council on Epidemiology and Prevention, Council on Functional Genomics and Translational Biology, and Council on Hypertension. Sex differences in the cardiovascular consequences of diabetes mellitus: a scientific statement from the American Heart Association. Circulation 2015;132(25):2424-2447

43 Chou AF, Scholle SH, Weisman CS, Bierman AS, Correa-de-Araujo R, Mosca L. Gender disparities in the quality of cardiovascular disease care in private managed care plans. Womens Health Issues 2007;17(3):120-130

44 Vaccarino V, Rathore SS, Wenger NK, et al; National Registry of Myocardial Infarction Investigators. Sex and racial differences in the management of acute myocardial infarction, 1994 through 2002. N Engl J Med 2005;353(7):671-682
45 Wenger NK. Coronary heart disease in women: highlights of the past 2 years-stepping stones, milestones and obstructing boulders. Nat Clin Pract Cardiovasc Med 2006;3(4):194-202

46 Randeva HS, Tan BK, Weickert MO, et al. Cardiometabolic aspects of the polycystic ovary syndrome. Endocr Rev 2012;33(5):812-841

47 Goueslard K, Cottenet J, Mariet AS, et al. Early cardiovascular events in women with a history of gestational diabetes mellitus. Cardiovasc Diabetol 2016;15:15

48 Gunderson EP, Chiang V, Pletcher MJ, et al. History of gestational diabetes mellitus and future risk of atherosclerosis in mid-life: the Coronary Artery Risk Development in Young Adults study. J Am Heart Assoc 2014;3(2):e000490

49 Yoon SS, Carroll MD, Fryar CD. Hypertension prevalence and control among adults: United States, 2011-2014. NCHS Data Brief 2015;(220):1-8

50 Sim JJ, Bhandari SK, Shi J, et al. Characteristics of resistant hypertension in a large, ethnically diverse hypertension population of an integrated health system. Mayo Clin Proc 2013;88(10):1099-1107

51 Mills KT, Bundy JD, Kelly TN, et al. Global disparities of hypertension prevalence and control: a systematic analysis of population-based studies from 90 countries. Circulation 2016;134(6):441-450

52 Nwankwo T, Yoon SS, Burt V, Gu Q. Hypertension among adults in the United States: National Health and Nutrition Examination Survey, 2011-2012. NCHS Data Brief 2013;(133):1-8

53 Grimm RH Jr, Grandits GA, Prineas RJ, et al; Treatment of Mild Hypertension Study (TOMHS). Long-term effects on sexual function of five antihypertensive drugs and nutritional hygienic treatment in hypertensive men and women. Hypertension 1997;29(1 Pt 1) :8-14

54 Divisón-Garrote JA, Ruilope LM, de la Sierra A, et al. Magnitude of hypotension based on office and ambulatory blood pressure monitoring: results from a cohort of 5066 treated hypertensive patients aged 80 years and older. J Am Med Dir Assoc 2017;18(5):452.e1-452.e6

55 Patel SA, Winkel M, Ali MK, Narayan KM, Mehta NK. Cardiovascular mortality associated with 5 leading risk factors: national and state preventable fractions estimated from survey data. Ann Intern Med 2015;163(4):245-253

56 Garovic VD, Bailey KR, Boerwinkle E, et al. Hypertension in pregnancy as a risk factor for cardiovascular disease later in life. J Hypertens 2010;28(4):826-833

57 Bokslag A, Teunissen PW, Franssen C, et al. Effect of early-onset preeclampsia on cardiovascular risk in the fifth decade of life. Am J Obstet Gynecol 2017;216(5):523.e1-523.e7

58 Celano CM, Daunis DJ, Lokko HN, Campbell KA, Huffman JC. Anxiety disorders and cardiovascular disease. Curr Psychiatry Rep 2016;18(11):101

59 Pimple P, Lima B, Hammadah M, et al. Psychological distress and subsequent cardiovascular events in individuals with coronary artery disease. J Am Heart Assoc 2019;8

60 Templin C, Ghadri JR, Diekmann J, et al. Clinical features and outcomes of Takotsubo (stress) cardiomyopathy. N Engl J Med 2015;373(10):929-938

61 Mosca L, Benjamin EJ, Berra K, et al; American Heart Association. Effectiveness-based guidelines for the prevention of cardiovascular disease in women-2011 update: a guideline from the American Heart Association. J Am Coll Cardiol 2011;57(12):1404-1423 